\title{
A randomized crossover study of the efficacy and safety of switching from insulin glargine to insulin degludec in children with type 1 diabetes
}

\author{
Tatsuhiko Urakami, Yusuke Mine, Masako Aoki, Misako Okuno and Junichi Suzuki
}

Department of Pediatrics, Nihon University School of Medicine, Tokyo, Japan

\begin{abstract}
This study implemented a randomized crossover design to evaluate the efficacy and safety of switching from insulin glargine (IGlar) to insulin degludec (IDeg) in 18 children (11 males, 7 females; age $11.0 \pm 0.5$ years) with type 1 diabetes. All subjects had previously used IGlar once daily at bedtime. We compared fasting plasma glucose (FPG) and $\mathrm{HbA} 1 \mathrm{c}$ levels, frequencies of overall and nocturnal $(2200 \mathrm{~h}-0659 \mathrm{~h})$ hypoglycemia, and basal insulin dose at the baseline with those measured during a 24-week period during which IGlar or IDeg was administered in combination with pre-meal rapid acting insulin analogues. IDeg was initially given at the same dose as IGlar but was subsequently titrated to achieve FPG levels of 90-140 mg/dL. There were no significant changes in FPG and HbAlc levels from the baseline during the 24-week study period with IGlar or IDeg. The daily basal insulin dose did not significantly differ with IGlar or IDeg. Although the frequencies of overall hypoglycemia were similar, nocturnal hypoglycemia significantly decreased at 12 and 24 weeks from the baseline with IDeg use ( $2 \pm 0.4$ vs. $0 \pm 0.3,0 \pm 0.5$ episodes/month, both $P<0.05)$, whereas no significant change in the frequency of nocturnal hypoglycemia was observed with IGlar. No severe hypoglycemia occurred during the study period with either basal insulin analogues. These results suggest that IDeg, injected once at bedtime, may provide similar glycemic control as IGlar while better reducing the risk of nocturnal hypoglycemia in children with type 1 diabetes.
\end{abstract}

Key words: Children, Glycemic control, Hypoglycemia, Insulin degludec, Type 1 diabetes

THE CLINICAL PRACTICE CONSENSUS GUIDELINES published by the International Society for Pediatric and Adolescent Diabetes (ISPAD) recommend insulin replacement as close to physiological levels as possible. In addition, the Guidelines state that optimal glycemic control without severe hypoglycemia must be the aim of insulin treatment for all children and adolescents with type 1 diabetes, regardless of age [1]. To attain this aim, basal-bolus insulin regimens, which involve multiple daily injections of insulin (MDI) or continuous subcutaneous insulin infusion with insulin analogues, are essential even for pediatric patients with type 1 diabetes. Patients on MDI regimens usually use a rapid-acting insulin analogue $(\mathrm{Ra})$ before meals as

Submitted Jun. 9, 2016; Accepted Sep. 12, 2016 as EJ16-0294 Released online in J-STAGE as advance publication Oct. 14, 2016 Correspondence to: Tatsuhiko Urakami, Department of Pediatrics, Nihon University School of Medicine, 1-6 Kandasurugadai, Chiyodaku, Tokyo 101-8309, Japan.

E-mail: urakami.tatsuhiko@nihon-u.ac.jp

Abbreviations: BMI, body mass index; FPG, fasting plasma bolus insulin regimen, in combination with a long-acting insulin analogue (La) once or twice daily as basal insulin regimen. This basal-bolus concept allows to adjust these insulin doses according to each patient's eating habits and lifestyles [1].

Despite the advantages of the basal-bolus insulin regimens with MDI, hypoglycemia, particularly nocturnal hypoglycemia, presents a major barrier in achieving desirable blood glucose levels. Some reports have shown that hypoglycemia has a injurious effect on central nervous system, particularly in young children. Fear of hypoglycemia can induce reluctance to titrate to blood glucose targets with sufficient insulin supplementation, thereby resulting in insufficient

glucose; HbA1c, glycated hemoglobin; IDeg, insulin degludec; IGlar, insulin glargine; IDet, inslin detemir; ISPAD, The International Society for Pediatric and Adolescent Diabetes; La, long acting insulin analogue; MDI, multiple daily injections of insulin; NGSP, National Glycohemoglobin Standardization Program; NPH, neutral protamine Hagedorn; PG, plasma glucose; $\mathrm{Ra}$, Rapid acting insulin analogue. 
glycated hemoglobin (HbA1c) levels that may progress to diabetic vascular complications [2]. The Diabetes Control and Complications Trial/Epidemiology of Diabetes Investigation and Complications (DCCT/ EDIC) studies have shown that suboptimal glycemic control during childhood and adolescence can increase the occurrence and development of diabetic vascular complications [3-5].

As mentioned above, basal-bolus regimens frequently comprise an $\mathrm{Ra}$ as a bolus regimen, and a $\mathrm{La}$, which has a longer action time as compared with neutral protamine Hagedorn (NPH) insulin, as a basal regimen [1]. Several reports have indicated that most patients using insulin glargine (IGlar) must administer more than twice-daily injections to cover their 24-hour basal insulin supplementation [6-8]. Insulin degludec (IDeg) is an ultra-La recently developed by Nordisk (Bagsvaerd, Denmark) that constitutes soluble multihexamers after subcutaneous injection; these molecules slowly dissociate and allow a slow and stable release of monomers into the circulation, and thus extending the action of basal insulin formula to more than 40 hours. In the phase 3 trials (BEGIN), IDeg has been shown further reduction in blood glucose levels in adult patients with type 1 or type 2 diabetes and further decrease in the frequencies of nocturnal hypoglycemia as compared with IGlar, although the occurrence of overall hypoglycemia did not differ significantly between the two Las [9-11]. Accordingly, IDeg is now widely used for adult patients with type 1 or type 2 diabetes in not only Japan but European Union and other countries.

Despite its popularity, only a few studies have reported the pharmacokinetics or clinical effect of IDeg in children and adolescents with type 1 diabetes. Biester et al. [12] demonstrated that the advantage of ultra-long pharmacokinetics of IDeg observed in adult patients were sustained in pediatric patients with type 1 diabetes. As for the clinical effect of IDeg in pediatric patients with type 1 diabetes, Thalange et al. [13] demonstrated the non-inferiority of IDeg to insulin detemir (IDet) with respect to improvement of HbAlc levels; however, the frequencies of overall and severe hypoglycemia were numerically higher with IDeg, despite numerically lower frequencies of nocturnal hypoglycemia with once-daily administration of IDeg. Furthermore, we recently reported improvement of glycemic control, assessed by fasting plasma glucose (FPG) and HbA1c levels and decrease in the frequencies of overall and nocturnal hypoglycemia, in young adults (15-35 years old) with type 1 diabetes after switching from IGlar to IDeg for a 1-year study period [14].

In the present study, we have compared the efficacy and safety of IGlar vs. IDeg as a basal-bolus therapy during sequential 24-week periods in a randomized crossover study of Japanese children with type 1 diabetes.

\section{Materials and Methods}

The study subjects comprised 18 Japanese children, 11 males and 7 females, with type 1 diabetes. The average age was $11.0 \pm 0.5$ (range: 7.2-14.2) years, and the average body mass index (BMI) was $16.0 \pm$ 1.5 (14.8-19.8), indicating non-obesity (BMI <25). Pubertal stage of the patients was at Tanner stage 1-3, and there was no growth abnormality in the patients. None of the patients had microvascular complications or psychosocial problems. In this group, the average FPG and HbAlc values, expressed according to the National Glycohemoglobin Standardization Program (NGSP) values, were $136 \pm 2 \mathrm{mg} / \mathrm{dL}$ and $7.8 \pm$ $0.2 \%$, respectively.

The subjects had been previously received a once-daily injection of IGlar at bedtime as a basal insulin regimen, in combination with pre-meal injections of $\mathrm{Ra}$, insulin aspart or insulin lispro as a pre-meal bolus insulin regimen. The average doses of IGlar and $\mathrm{Ra}$ were $0.45 \pm 0.20$ units $/ \mathrm{kg} / \mathrm{day}$ and $0.55 \pm 0.30$ units/ $\mathrm{kg} /$ day, respectively, although the latter baseline was adjusted by using carbohydrate counting. Subjects self-monitored plasma glucose (PG) levels daily before each meal, at bedtime, and whenever symptoms of hypoglycemia were recognized.

After obtaining informed consent from the patients as well as their parents, the subjects were randomly divided into two groups. Nine subjects switched to an insulin regimen comprising once-daily injection of IDeg at bedtime for 24 weeks in combination with pre-meal Ra, and thereafter injected IGlar for the next 24 weeks after a 1-week stabilization period (Group A). The other 9 subjects continued to inject IGlar as a basal insulin for 24 weeks, and thereafter switched to a once-daily injection of IDeg at bedtime for the next 24 weeks after a 1-week stabilization period (Group B) (Fig. 1). The characteristics of patients in Groups A and $\mathrm{B}$ are shown in Table 1. 


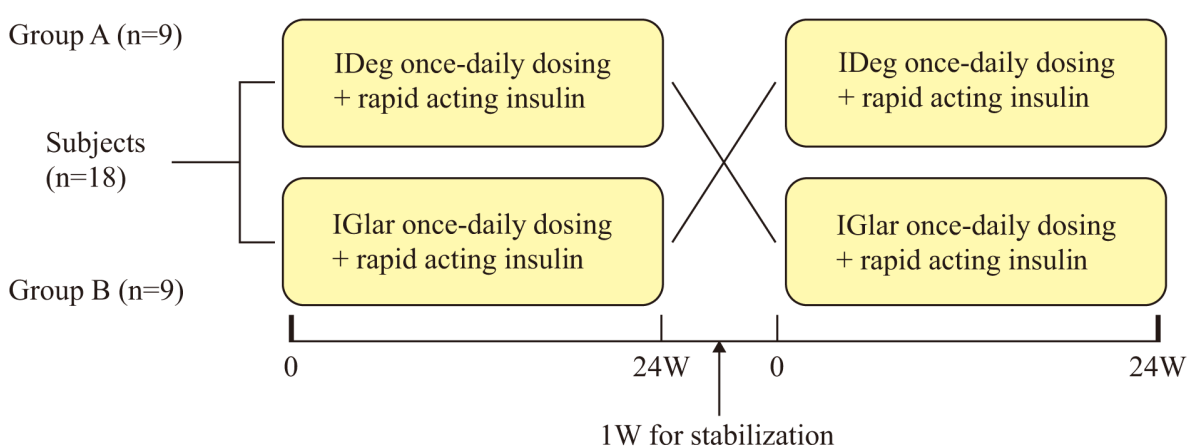

Fig. 1 Randomized crossover study design

IDeg, insulin degludec; IGlar, insulin glargine. Randomization: Group A: 24W IDeg $\rightarrow$ 24W IGlar, Group B: 24W IGlar $\rightarrow$ 24W IDeg.

Table 1 Subjects characteristics in Groups A and B

\begin{tabular}{lccc}
\hline & $\begin{array}{c}\text { Total } \\
(\mathrm{n}=18)\end{array}$ & $\begin{array}{c}\text { Group A } \\
(\mathrm{n}=9)\end{array}$ & $\begin{array}{c}\text { Group B } \\
(\mathrm{n}=9)\end{array}$ \\
\hline $\mathrm{M} / \mathrm{F}$ & $11 / 7$ & $6 / 3$ & $5 / 4$ \\
Age $(\mathrm{yr})$ & $11 \pm 0.5$ & $10 \pm 0.5$ & $11 \pm 0.5$ \\
FPG (mg/dL) & $136 \pm 2$ & $134 \pm 3$ & $138 \pm 4$ \\
HbA1c (\%) & $7.8 \pm 0.2$ & $7.7 \pm 0.3$ & $7.8 \pm 0.3$ \\
\hline
\end{tabular}

The results are expressed as medians \pm standard errors.

We initially set the dose of IDeg to be the same dose as IGlar when changed, but were then titrated to achieve self-monitored FPG levels of $90-140 \mathrm{mg} /$ dL. Subjects were requested to sustain self-monitoring of PG before meals and at bedtime, as well as whenever diurnal or nocturnal symptoms of hypoglycemia were noted. Hypoglycemia was defined as a self-monitored PG level $<70 \mathrm{mg} / \mathrm{dL}$, and hypoglycemia occurring between $2200 \mathrm{~h}-0659 \mathrm{~h}$ was classified as nocturnal. Nocturnal hypoglycemia was defined as when the subject noted symptoms of hypoglycemia with self-monitored PG levels $<70 \mathrm{mg} / \mathrm{dL}$. In children, severe hypoglycemia is defined as an event associated with impaired consciousness or seizure, whereas in adults, only an event requiring assistance from another person is defined as severe. However, toddlers usually need assistance to correct even mild to moderate hypoglycemia [2].

In the present study, we compared the FPG and HbA1c levels, frequencies of overall and nocturnal hypoglycemia, and basal insulin doses at the baseline with those at the 4th, 12th, and 24th week when using IGlar or IDeg in combination with pre-meal
Ras through a randomized crossover study. BMI and problematic adverse events, including occurrence of severe hypoglycemia, were also evaluated. Each patient self-monitored FPG at home using a glucometer (One Touch Ultra; Johnson \& Johnson, New Brunswick, NJ, USA). HbAlc was measured at the Nihon University Hospital laboratory using a high performance liquid chromatography method (4.6$6.1 \%$ : reference value).

\section{Statistical analyses}

Results were presented as medians \pm standard errors (SEs). The Wilcoxon signed-rank test was used to evaluate statistical differences between the two results. A one-way repeated measures analysis of variance (ANOVA) with the Greenhouse-Geisser correction was used to evaluate statistical differences in median values, and post-hoc multiple comparisons were used to evaluate changes of median values from the baseline by a Dunnett's method. Statistical significant was accepted at $P$-value $<0.05$. All statistical analyses were conducted by IBM SPSS Statistics, version 23.0 (IBM Corp, Armonk, NY, USA). 


\section{Results}

Comparisons of FPG and HbAlc levels and basal insulin doses at the baseline with those at the 4th, 12th, and 24th week when using IDeg or IGlar

FPG and HbA1c levels at baseline and those at 4th, 24th, and 48th weeks after receiving IDeg or IGlar once-daily at bedtime were compared (Table 2). There were no significant changes in FPG and $\mathrm{HbAlc}$ values at 4th, 12th, or 24th weeks from the baseline with either IGlar or IDeg. Similarly, the daily basal doses of either analogue did not significantly differ during the 24-week study period relative to the baseline. In addition, no significant changes were observed in the daily bolus insulin dose, although this was adjusted based on carbohydrate counting during the study period (data not shown).

Comparisons of the frequencies of overall and nocturnal hypoglycemia events at baseline with those at the 4th, 12th, and 24th week when using IDeg or IGlar

The frequencies of overall hypoglycemia were similar with the two basal insulin analogues throughout the 24-week study period (the overall hypoglycemia rates numerically decreased with IDeg, but not significant), whereas nocturnal hypoglycemia (between $2200 \mathrm{~h}-$ $0659 \mathrm{~h}$ ) significantly decreased at the 12th and 24th week relative to the baseline with IDeg $(2 \pm 0.4$ for the baseline vs. $0 \pm 0.3$ for the 12th week, $0 \pm 0.5$ for the 24th week episodes/month, both $P<0.05$ ), whereas there was no significant difference in the frequency of nocturnal hypoglycemia events with IGlar (Table 3). No severe hypoglycemia occurred at any time throughout the study period with either basal insulin analogue. In addition, BMI did not change significantly during the study period with either basal insulin analogue (data not shown). Excluding mild hypoglycemia, there were no problematic adverse events associated with use of IDeg at any time throughout the study period.

\section{Discussion}

The ISPAD has proposed a target HbA1c of $<7.5 \%$ for all children and adolescents with type 1 diabetes, regardless of age [15], with the intent that this glycemic target will prevent diabetic vascular complications while also avoiding frequent hypoglycemia and

Table 2 Comparisons of FPG and HbA1c levels and basal insulin doses at the baseline with those at the 4th, 12th, and 24th week when using IDeg or IGlar

\begin{tabular}{lcccc}
\hline & 0 & $4 \mathrm{~W}$ & $12 \mathrm{~W}$ & $24 \mathrm{~W}$ \\
\hline FPG: IGlar (mg/dL) & $134 \pm 3$ & $136 \pm 3$ & $135 \pm 3$ & $135 \pm 2$ \\
FPG: IDeg (mg/dL) & $138 \pm 4$ & $136 \pm 4$ & $135 \pm 3$ & $134 \pm 4$ \\
HbA1c: IGlar (\%) & $7.7 \pm 0.3$ & $7.8 \pm 0.2$ & $7.9 \pm 0.3$ & $7.8 \pm 0.2$ \\
HbA1c: IDeg (\%) & $7.8 \pm 0.3$ & $7.9 \pm 0.3$ & $7.8 \pm 0.2$ & $7.8 \pm 0.2$ \\
BID: IGlar (U/kg/day) & $0.45 \pm 0.18$ & $0.46 \pm 0.18$ & $0.48 \pm 0.15$ & $0.48 \pm 0.15$ \\
BID: IDeg (U/kg/day) & $0.46 \pm 0.20$ & $0.44 \pm 0.15$ & $0.44 \pm 0.14$ & $0.43 \pm 0.15$ \\
\hline
\end{tabular}

FPG, fasting plasma glucose; HbAlc, glycated hemoglobin; IDeg, insulin degludec; IGlar, insulin glargine; BID, basal insulin dose. The results are expressed as medians \pm standard errors.

Table 3 Comparisons of the frequencies of overall and nocturnal hypoglycemia events at the baseline with those at the 4th, 12th, and 24th week when using IDeg or IGlar

\begin{tabular}{lcccc}
\hline & 0 & $4 \mathrm{~W}$ & $12 \mathrm{~W}$ & $24 \mathrm{~W}$ \\
\hline Total hypo: IGlar (time/month) & $5 \pm 0.4$ & $5 \pm 0.5$ & $5 \pm 0.5$ & $4 \pm 0.7$ \\
Total hypo: IDeg (time/month) & $6 \pm 0.6$ & $4 \pm 0.5$ & $3 \pm 0.5$ & $3 \pm 0.6$ \\
Nocturnal hypo: IGlar (time/month) & $2 \pm 0.5$ & $2 \pm 0.5$ & $2 \pm 0.4$ & $2 \pm 0.4$ \\
Nocturnal hypo: IDeg (time/month) & $2 \pm 0.5$ & $1 \pm 0.5$ & $0 \pm 0.3 *$ & $0 \pm 0.5 *$ \\
\hline
\end{tabular}

* $P<0.05$ vs. baseline. IDeg, insulin degludec; IGlar, insulin glargine; Hypo, hypoglycemia. The results are expressed as medians \pm standard errors. 
the occurrence of severe hypoglycemia. The avoidance of hypoglycemic episodes, which can cause various brain functions, should be given the highest priority in pediatric patients, particularly young children [2]. In a basal-bolus insulin regimen, the ideal bolus preparation would synchronize the post-prandial PG rise over the short term, whereas the ideal basal-insulin preparation would show stable pharmacodynamics with a less peak and minimal day-to-day variations. Such a basal-bolus regimen could be used to achieve appropriate glycemic control (i.e., target $\mathrm{HbA} 1 \mathrm{c}$ $<7.5 \%$ ) while minimizing hypoglycemia in pediatric patients of all ages [5].

IDeg might represent a desirable La alternative to IGlar for patients who cannot achieve optimal glycemic control despite careful Ra dose adjustments based on carbohydrate counting. Several studies in adults have shown that IDeg exhibits the desired optimal pharmacokinetic properties and confers a low risk of hypoglycemia in patients with both type 1 and type 2 diabetes $[9-11,16]$. In addition, the majority of the studies have reported comparable glucose-lowering effects of IDeg and IGlar in patients with type 1 diabetes, as demonstrated by equivalent FPG and HbA1c levels [9, 13, 17-19]. As mentioned in the introduction, in a 26-week study of pediatric patients with type 1 diabetes of all ages, Thalange et al. [13] reported the non-inferiority of IDeg as compared with IDet in regard with changes in $\mathrm{HbA} 1 \mathrm{c}$ levels from the baseline. On the other hand, we previously demonstrated an improvement in glycemic control in young adults with type 1 diabetes after changing from IGlar to IDeg [14]. We therefore considered the potential for well-responders and lwss-responders to IDeg as a basal insulin preparation, although most patients exhibited significant improvements in FPG and HbA1c levels and decreases in frequencies of hypoglycemia after changing from IGlar to IDeg, which might have considerably influenced the desired results [14].

In the present study, we did not observe a significant improvement in glycemic control, assessed from changes in FPG and HbAlc levels vs. the baseline after changing from IGlar to IDeg. The possible reasons for this difference in results of the present pediatric study and those of our previous study of young adults include the following: first, the PG profiles in young children might be more strongly affected by bolus insulin regimens than by basal insulin regimens, as the amounts of food consumption and physical exercise are much less predictable in children than in adults. In general, it is difficult to adjust the bolus insulin doses to achieve optimal post-prandial glycemia in children with type 1 diabetes, even when using the carbohydrate counting method [1]. Accordingly, it is important to adjust the bolus insulin doses using adequate insulin preparations in order to achieve optimal glycemic control in pediatric patients [20]. Second, children are likely to lack experience with injection skills and techniques; some are unable to maintain insulin injection schedules, and others may not achieve correct injection-site rotation and/or subcutaneous injection depths, which can influence insulin absorption and effectiveness [21]. Third, young adults were better able to reinforce their attitudes towards aspects of diabetes self-management, which include the frequency of PG self-monitoring and maintenance of an appropriate lifestyle, during study periods involving new insulin preparations. Therefore, young adults might be better able to achieve desirable glycemic control, compared with children and the elderly.

We noted similar frequencies of overall hypoglycemia with the two basal insulin analogues (overall hypoglycemia rates numerically decreased with IDeg, but not significant) during the 24-week study period, in contrast to a significant decrease in nocturnal hypoglycemia from the baseline with IDeg but not with IGlar. Moreover, some children who used IDeg experienced no events of hypoglycemia throughout the study period. The 2-year extension of the main BEGIN trial [22] indicated that the hypoglycemia-reducing benefits of IDeg were observed at night, rather than during the day; this is likely because a relationship exists between bolus insulin and diurnal hypoglycemia, whereas the ultra-long acting and less variable glucose-lowering effects of IDeg may better decrease nocturnal hypoglycemia. Some other studies [9, 13, 17-19, 23-26], including our recent study [14], also demonstrated a greater reduction in the risk of nocturnal hypoglycemia, rather than an overall decrease, with IDeg in all-aged patients with type 1 diabetes. The outcomes of an earlier 1-year study of IDeg in Japanese adults with type 2 diabetes were consistent with our observations [11]. Reduction of nocturnal hypoglycemia is considered to be caused by lower pharmacodynamic variability of IDeg than IGlar during nighttime [16]. Biester $\mathrm{T}$ et al. [11] reported that the area under the blood concentration-time curve of IDeg in children was greater than adults (not statistically significant), 
which does not seem to influence a risk of nocturnal hypoglycemia in the present study.

Nocturnal hypoglycemia can cause anxiety and morbidity for the family members of children with type 1 diabetes [27], particularly as children with type 1 diabetes are much less likely to be awakened by hypoglycemia than are children without diabetes because the counterregulatory response to hypoglycemia is attenuated during nighttime [28]. Recent study has reported a high prevalence (up to $40 \%$ ) of prolonged nocturnal hypoglycemia events during nighttime in pediatric patients with type 1 diabetes [2]. Such events may induce seizures and, occasionally, death. Therefore, the prevention of nocturnal hypoglycemia should be the most important matter in particularly the management of children and adolescents with type 1 diabetes. Consequently, the most valuable benefit of IDeg might be the reduction in nocturnal hypoglycemia in patients with type 1 diabetes, regardless of age.

We note that patients should be made aware that the half-life and action time of IDeg might be different from those of other La formulations. These differences in formulations should be considered to minimize the risk of developing hypoglycemia. Our previous study in young adults with type 1 diabetes recommended to begin replacement of IDeg at a 10-20\% lower dose when IGlar was previously injected once daily, or at a 20-30\% lower dose when IGlar was injected twice daily [14]. In the present study, the daily dose of IDeg was numerically decreased, but did not significantly differ during the 24-week study period relative to the baseline; therefore, the administration of a conservative dose is recommended in children of age $<15$ years with type 1 diabetes upon switching from IGlar previously given once daily to IDeg. Subsequently, this dose can be adjusted according to the patient's blood glucose levels to avoid occurrence of hypoglycemia [9, 11, 18, 19, 24-26]. The dose-adjustment could affect a risk of hypoglycemia.

Some limitations of the present study should be acknowledged. First, the inclusion of a small number of subjects among which to evaluate the efficacy and safety of IDeg limited the amount of data collected, particularly as for safety. In the present study, we did not find no problematic adverse events associated with using IDeg, such as occurrence of severe hypoglycemia; however, such adverse events could occur in a larger number of subjects. Given the diffi- culty in recruiting a large number of participants for a single-institution crossover study, a multi-center study is therefore needed to confirm our findings. In addition, it may be necessary to evaluate the efficacy and safety of IDeg in younger children aged below 7 years. Second, switching to a new insulin product might introduce bias related to patients' attitudes towards the self-care of diabetes and maintenance of appropriate lifestyles, which might alter the outcome of the study. In addition, the investigators might be more vigilant in the diabetes care when using a new insulin preparation. These factors might all have affected the outcome of the study.

\section{Conclusions}

In the present study, we observed the non-inferiority of glycemic control, as assessed by measuring FPG and $\mathrm{HbA} 1 \mathrm{c}$ levels, in children with type 1 diabetes after changing from IGlar to IDeg. The frequencies of overall hypoglycemia were similar in the two basal insulin analogues, whereas nocturnal hypoglycemia significantly decreased at the 12th and 24th week from the baseline with IDeg; in contrast, no significant change in nocturnal hypoglycemia was observed with IGlar. No severe hypoglycemia occurred with either basal insulin analogue during the study period. These results suggest that IDeg, injected once daily at bedtime, provides similar glycemic control to IGlar while reducing the risk of nocturnal hypoglycemia. Optimal glycemic targets and the minimization of hypoglycemia might be well achieved with a basal-bolus therapy that includes IDeg in children with type 1 diabetes.

\section{Acknowledgements}

This study was approved by the ethics committee of Nihon University School of Medicine (NO.140202) and was performed in accordance with the ethical standards set forth in the 1964 Declaration of Helsinki and its later amendments.

\section{Disclosure Statement}

T.U. received honoraria from Sanofi and Novo Nordisk as a speaker and for attendance at advisory boards. Other authors have no conflicts of interest. 


\section{References}

1. Danne T, Bangstad H-J, Deeb L, Jarosz-Chabot P, Mungaie L, et al. (2014) ISPAD Clinical Practice Consensus Guidelines 2014. Insulin treatment in children and adolescents with diabetes. Pediatr Diabetes 15 (Suppl 20): 115-134.

2. Ly TT, Maahs DM, Rewers A, Dunger D, Oduwole A, et al. (2014) ISPAD Clinical Practice Consensus Guidelines 2014. Assessment and management of hypoglycemia in children and adolescents with diabetes. Pediatr Diabetes 15 (Suppl 20): 180-192.

3. (1993) The effect of intensive treatment of diabetes on the development and progression of long-term complications of insulin-dependent diabetes mellitus. The Diabetes Control and Complications Trial Research Group. N Engl J Med 329: 977-986.

4. Writing Team for the Diabetes Control and Complications Trial Research Group/Epidemiology of Diabetes Interventions and Complications Research Group (2002) Effect of intensive therapy on the microvascular complications of type 1 diabetes mellitus. JAMA 287: 2563-2569.

5. Nathan DM, Cleary PA, Backlund JY, Genuth SM, Lachin JM, et al. (2005) Intensive diabetes treatment and cardiovascular disease in patients with type 1 diabetes. N Engl J Med 353: 2643-2653.

6. Porcellati F, Rossetti P, Busciantella NR, Marzotti S, Lucidi P, et al. (2007) Comparison of pharmacokinetics and dynamics of the long-acting insulin analogs glargine and detemir at steady state in type 1 diabetes: a double-blind, randomized, crossover study. Diabetes Care 30: 2447-2452.

7. Urakami T, Kuwabara R, Habu M, Komiya K, Nagano $\mathrm{N}$, et al. (2012) Comparison of the injection frequencies employed and basal-to-total insulin dose ratios obtained when glargine and detemir are used in children with type 1 diabetes mellitus treated by basal-bolus therapy. Diabetol Int 3: 75-79.

8. Urakami T, Naito Y, Seino Y (2014) Insulin glargine in pediatric patients with type 1 diabetes in Japan. Pediatr Int 56: 822-828.

9. Heller S, Buse J, Fisher M, Garg S, Marre M, et al. (2012) Insulin degludec, an ultra-longacting basal insulin, versus insulin glargine in basal-bolus treatment with mealtime insulin aspart in type 1 diabetes (BEGIN Basal-Bolus Type 1): a phase 3, randomized, openlabel, treat-to-target non-inferiority trial. Lancet 379 : 1489-1497.

10. Garber AJ, King AB, Del Prato S, Sreenan S, Balci MK, et al. (2012) Insulin degludec, an ultra-longacting basal insulin, versus insulin glargine in basal-bolus treatment with mealtime insulin aspart in type 2 diabetes (BEGIN Basal-Bolus Type 2): a phase 3, randomized, openlabel, treat-to-target non-inferiority trial. Lancet 379 :
1498-1507.

11. Kaku K, Eid MA (2015) Safety, efficacy, and early clinical experience of insulin degludec in Japanese people with diabetes mellitus: A first-year report from Japan. $J$ Diabetes Investig 6: 610-619.

12. Biester $\mathrm{T}$, Blaesig $\mathrm{S}$, Remus $\mathrm{K}$, Aschemeier $\mathrm{B}$, Kordonouri O, et al. (2014) Insulin degludec's ultralong pharmacokinetic properties observed in adults are retained in children and adolescents with type 1 diabetes. Pediatr Diabetes 15: 27-33.

13. Thalange $\mathrm{N}$, Deeb L, Iotova V, Kawamura $\mathrm{T}$, Klingensmith G, et al. (2015) Insulin degludec in combination with bolus insulin aspart is safe and effective in children and adolescents with type 1 diabetes. Pediatr Diabetes 16: 164-176.

14. Urakami T, Kuwabara R, Aoki M, Okuno M, Suzuki J (2016) Efficacy and safety of switching from insulin glargine to insulin degludec in young people with type 1 diabetes. Endocr J 63: 159-167.

15. Rewers MJ, Pillay K, de Beaufort C, Craig ME, Hanas $\mathrm{R}$, et al. (2014) Assessment and monitoring of glycemic control in children and adolescents with diabetes. Pediatr Diabetes 15 Suppl 20: 102-114.

16. Heise T, Hermanski L, Nosek L, Feldman A, Rasmussen $\mathrm{S}$, et al. (2012) Insulin degludec: four times lower pharmacodynamic variability than insulin glargine under steady-state conditions in type 1 diabetes. Diabetes Obes Metab 14: 859-864.

17. Ratner RE, Gough SC, Mathieu C, Del Prato S, Bode B, et al. (2013) Hypoglycemia risk with insulin degludec compared with insulin glargine in type 2 and type 1 diabetes: a pre-planned meta-analysis of phase 3 trials. Diabetes Obes Metab 15: 175-184.

18. Birkeland KI, Home PD, Wendisch U, Ratner RE, Johansen T, et al. (2011) Insulin degludec in type 1 diabetes: a randomized controlled trial of a new-generation ultra-long-acting insulin compared with insulin glargine. Diabetes Care 34: 661-665.

19. Bode BW, Buse JB, Fisher M, Garg SK, Marre M, et al. (2013) Insulin degludec improves glycemic control with lower nocturnal hypoglycemia risk than insu-

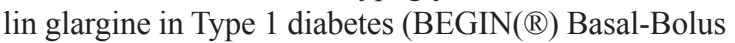
Type 1): 2-year results of a randomized clinical trial. Diabet Med 30: 1293-1297.

20. Urakami T, Kuwabara R, Habu M, Okuno M, Suzuki J, et al. (2015) Efficacy and safety of switching to insulin glulisine from other rapid-acting insulin analogues in children with type 1 diabetes. $J$ Diabetes Investig 6: 87-90.

21. Bantle JP, Neal L, Frankamp LM (1993) Effects of the anatomical region used for insulin injections on glycemia in type 1 diabetes subjects. Diabetes Care 16: 1592-1597. 
22. Bode BW, Buse JB, Fisher M, Garg SK, Marre M, et al. (2013) Insulin degludec improves glycemic control with lower nocturnal hypoglycemia risk than insulin glargine in Type 1 diabetes (BEGIN(®) Basal-Bolus Type 1): 2-year results of a randomized clinical trial. Diabet Med 30: 1293-1297.

23. Yamada K, Nakayama H, Sato S, Tajiri Y, Kaku H, et al. (2014) A randomized crossover study of the efficacy and safety of switching from insulin glargine to insulin degludec among patients with type 1 diabetes. Diabetol Int 5: 74-77.

24. Kusunoki Y, Katsuno T, Miyakoshi K, Ikawa T, Nakae $\mathrm{R}$, et al. (2013) Effects of switching from insulin degluudec in patients with type 1 diabetes mellitus. Diabetes Ther 4: 461-472.

25. Nakamura T, Sakaguchi K, So A, Nakajima S, Takabe
M, et al. (2015) Effects of insulin degludec and insulin glargine on day-to-day fasting plasma glucose variability in individuals with type 1 diabetes: a multicenter, randomized, crossover study. Diabetologia 58: 2013-2019.

26. Yamamoto C, Miyoshi H, Fujiwara Y, Kameda R, Ichiyama M, et al. (2016) Degludec is superior to glargine in terms of daily glycemic variability in people with type 1 diabetes mellitus. Endocr J 63: 53-60.

27. Monaghan MC, Hilliard ME, Cogen FR, Streisand R (2009) Nighttime caregiving behaviors among parents of young children with type 1 diabetes: association with illness characteristics and parent functioning. Fam Syst Health 27: 28-38.

28. Jones TW, Porter P, Sherwin RS, Davis EA, O'Leary P, et al. (1998) Decreased epinephrine responses to hypoglycemia during sleep. $N$ Engl J Med 338: 1657-1662. 